\title{
First 3-D Reconstructions of Targets Hidden Beneath Foliage by Means of Polarimetric SAR Tomography
}

\author{
Matteo Nannini, Rolf Scheiber, Ralf Horn, and Alberto Moreira, Fellow, IEEE
}

\begin{abstract}
SAR tomography (SARTom) is an imaging technique that allows multiple phase center separation in the vertical direction, leading to a 3-D reconstruction of the imaged scene. The retrieval of volume structure information (e.g., for forest classification) and the solution of the layover problem are two of the most promising applications. In this letter, SARTom, in combination with polarimetry (PolSARTom), is exploited to image and to extract characteristic features (e.g., shape and height) of targets hidden beneath foliage. This analysis is applied to L-band airborne data acquired by the E-SAR system of the German Aerospace Center (DLR) during a tomographic campaign that took place in September 2006 on the test site of Dornstetten (Germany).
\end{abstract}

Index Terms-Polarimetry, SAR tomography (SARTom), superresolution, target detection.

\section{INTRODUCTION}

$\mathbf{S}$ AR tomography (SARTom) makes it possible to obtain a complete 3-D representation of the scene. The first demonstration of airborne SARTom was presented in [1], and the main constraints in terms of resolution and ambiguity rejection were analyzed. When the focusing step is performed by means of the coherent Fourier beamformer, realistic working conditions such as nonuniform track distribution can heavily impact the final results. This is why, in recent years, regularized linear inversion [2] and modern beamforming techniques such Capon [3] and MUSIC [4] were introduced to allow higher ambiguity rejection and improvements upon the Fourier resolution [5], [6]. Despite these drawbacks, the advantage of the linear beamformer is that its final resolution can be predicted, and it maintains the signal phase due to its coherent nature. Because of this, it is usually used as a reference in order to plan the acquisition geometry. In this case, the relation between the height resolution $\rho$ in the perpendicular line-of-sight (PLOS) direction and tomographic aperture dimension $L_{\text {tomo }}$ is

$$
\rho=\frac{\lambda r_{0}}{2 L_{\text {tomo }}}
$$

where $r_{0}$ is the master slant range distance. In order to avoid ambiguities within maximum volume height $V$, average base-

Manuscript received July 20, 2010; revised December 15, 2010 and March 17, 2011; accepted April 3, 2011.

The authors are with the SAR Technology Department, Microwaves and Radar Institute, German Aerospace Center (DLR), 82234 Wessling, Germany (e-mail: matteo.nannini@dlr.de).

Digital Object Identifier 10.1109/LGRS.2011.2160329 line $d$ is constrained by

$$
d \leq \frac{\lambda r_{0}}{2 V}
$$

Combining (1) and (2), the required number of tracks is $N=\left(L_{\text {tomo }} / d\right)+1$. These relations were used in the planning of a tomographic campaign that took place in Dornstetten (Germany). Its goal was to analyze the potential of SARTom for extracting information concerning targets hidden beneath the foliage. For this experiment, an average tomographic resolution of $2 \mathrm{~m}$ and a maximum volume height of around $30 \mathrm{~m}$ were anticipated.

Due to the penetration depth of long-wavelength radars (e.g., at L- and P-bands), a vegetated scenario represents a particularly interesting application of SARTom because, in principle, the actual distribution of the scatterers along the PLOS can be retrieved. Because of this, several investigations based on direct, as well as model-based, inversion of the tomographic data have been carried out (see [7] and [8], respectively).

Moreover, the exploitation and potential of SAR to penetrate foliage (FOPEN) is a topic of great relevance in remote sensing for defense applications [9].

The objective of this letter is twofold, i.e., to present the first tomographic results concerning targets hidden beneath foliage and to perform a comparison between tomograms obtained in different polarization bases in order to analyze how polarimetry can enhance the target signature in comparison to the canopy to allow the retrieval of the height of the hidden targets.

\section{SARTom PROCESSING}

Once the acquired raw data are processed with the extended chirp scaling algorithm [10], including corrections due to the topography [11], they are coregistered and are ready to be processed by a beamforming technique coupled with a heightdependent motion compensation and coregistration approach [12]. The so-called steering vector $\mathbf{a}(h)$ is defined as

$$
\mathbf{a}(h)=\exp \left(j \frac{4 \pi}{\lambda} \mathbf{R}_{h}\right)
$$

with $\mathbf{R}_{h}=\left[R_{1}(h), \ldots, R_{N}(h)\right]$ representing the sensor-totarget distance vector for height $h$. After the sample complex covariance matrix $\mathbf{C}$ has been computed, the Capon beamformer and the MUSIC algorithm can be applied. The form of the resulting responses (power for Capon and pseudopower for MUSIC) can be written as

$$
\mathbf{P}(h)=\frac{1}{\mathbf{a}^{*}(h) \mathbf{X} \mathbf{a}(h)}
$$




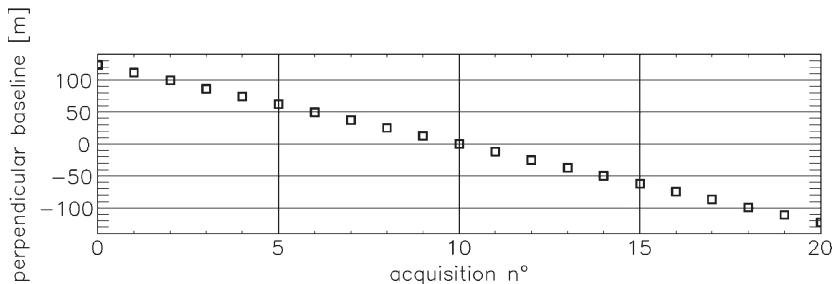

Fig. 1. Perpendicular baselines' distribution at midrange (look angle of $45^{\circ}$ ) for the Dornstetten tomographic experiment, which is depicted in the plane perpendicular to the flight direction.

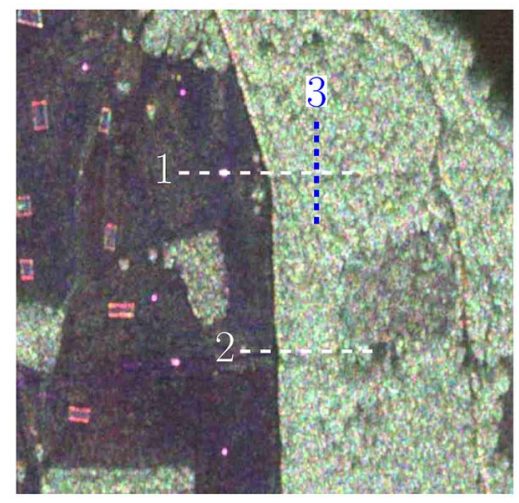

Fig. 2. Full polarimetric SLC image $(600 \mathrm{~m} \times 600 \mathrm{~m})$. Coding: RGB $(\mathrm{HH}$, HV, and VV). (Dashed lines) The three profiles, along which tomographic processing is carried out, are depicted. Azimuth is horizontal, and range is vertical.

where $*$ indicates the complex conjugate transpose, and $\mathbf{X}$ depends on whether the Capon beamforming or the MUSIC algorithm is applied. For Capon and MUSIC, $\mathbf{X}=\mathbf{C}^{-1}$ or $\mathbf{X}=\mathbf{E}_{\mathbf{N}} \mathbf{E}_{\mathbf{N}}^{*}$, respectively. $\mathbf{E}_{\mathbf{N}}$ is the matrix of eigenvectors associated with the noise subspace of $\mathbf{C}$. By scanning the image stack in the azimuth and range direction, a 3-D intensity reconstruction as a function of height (a tomogram) can be obtained. Selecting different polarization channels and bases, it is now possible to produce tomograms as a function of the polarization [1].

\section{EXPERIMENT}

The data set was acquired close to Dornstetten (Germany) at L-band in September 2006. Some targets of interest (e.g., vehicles, containers, and corner reflectors) were located inside and outside the forest in order to evaluate the impact of the canopy on the target response. The area where the experiment took place is relatively flat, and half of the region is covered by inhomogeneous forest stands of different species. The tree height varies between 10 and $30 \mathrm{~m}$.

The acquisition geometry is a regular horizontal grid of 21 tracks with an average baseline of $20 \mathrm{~m}$. The actual acquisition geometry is very close to the planned one, with a maximum deviation of around $4 \mathrm{~m}$ between the nominal and real tracks. The orthogonal baselines for the midrange, corresponding to the location of the hidden truck, are reported in Fig. 1. Fig. 2 illustrates the relevant part of the fully polarimetric single-look complex (SLC) image in the RGB coding (HH, HV, and VV). The tomographic processing results presented in the following were obtained along the cuts indicated in the image. Profile 1 of Fig. 2 includes two trucks, i.e., one is located outside and the other inside the forest, and profile 2 includes a hidden container.

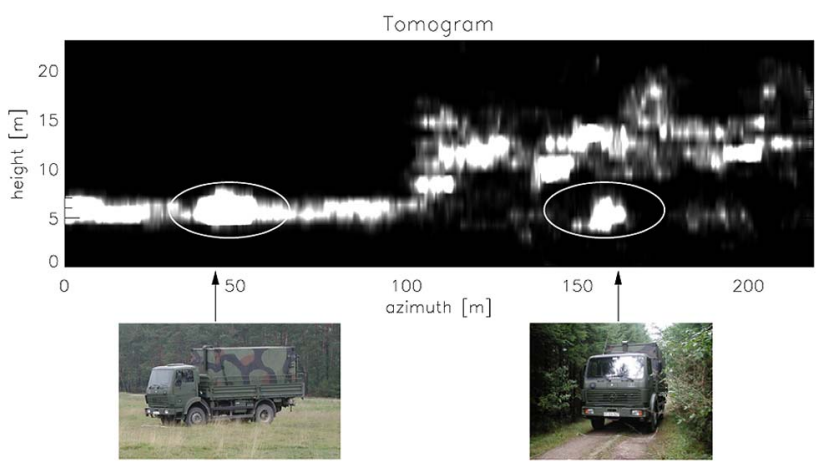

Fig. 3. Tomogram (slice along azimuth) in the $\mathrm{HH}$ polarization representing two trucks, i.e., one outside and the other inside the canopy. The tomographic reconstruction in the PLOS is displayed as true height.

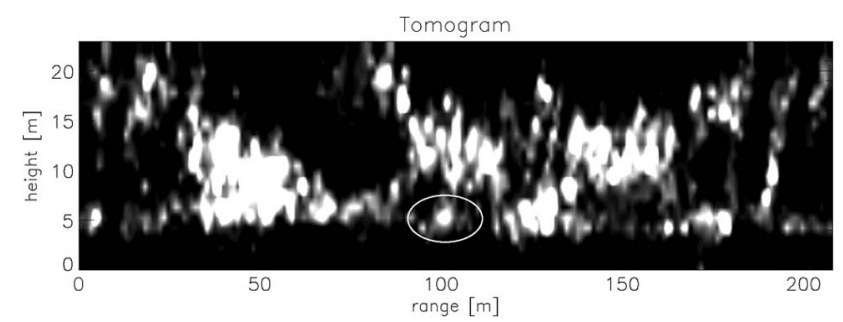

Fig. 4. Tomogram (slice along range) in the $\mathrm{HH}$ polarization representing the hidden truck (ellipse). The tomographic reconstruction in the PLOS is resampled (shift as a function of height) to allow correct representation in the vertical dimension.

It is worth mentioning that the hidden truck is located on a path in the forest and, therefore, there is no vegetation directly above it. However, in the radar side-looking geometry, the truck can be considered as hidden since there is a layover of the canopy in the resolution cell containing the truck.

\section{POLARIMETRIC SARTom ANALYSiS}

First, tomograms obtained by means of the Capon beamformer will be presented for the $\mathrm{HH}$ channel. Then, the impact of polarization will be examined by changing the polarization channel and the polarimetric basis. Results obtained by means of a coherent Fourier beamformer, as well as with the MUSIC algorithm, will conclude this section. For the superresolution methods, the sample covariance matrix has been computed, including a diagonal load and using 25 snapshots.

\section{A. HH Polarization}

Fig. 3 illustrates the tomogram related to the trucks. This profile represents the layover solution in the PLOS direction, which was then projected to the vertical axis to associate the scatterers with their actual height. As indicated in the figure, one spot represents the truck outside the forest while another is associated with the one inside it. The canopy over the second truck is also clearly visible. The absence of the ground under the canopy is due to the fact that the ground-trunk double-bounce reflection is missing because the truck has been placed on a small track inside the forest, parallel to the flight direction, on which no trees were present. Without these reflections, the backscattered power related to a terrain contribution is much smaller than the one from the canopy or the hidden target. Let us now analyze Fig. 4, which represents the tomographic reconstruction along profile 3 of Fig. 2. In this ground-range 


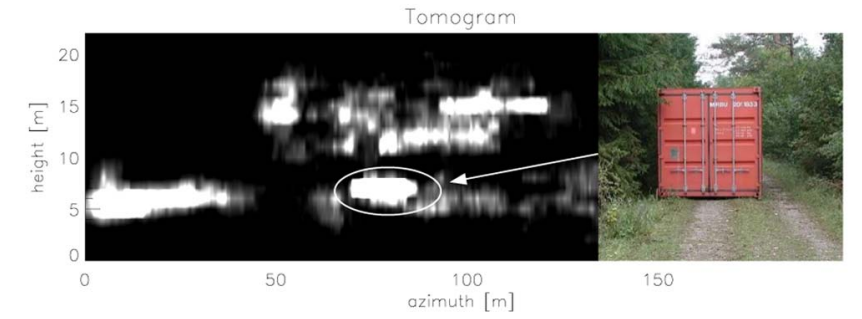

Fig. 5. Tomogram in the $\mathrm{HH}$ polarization representing the container inside the forest.
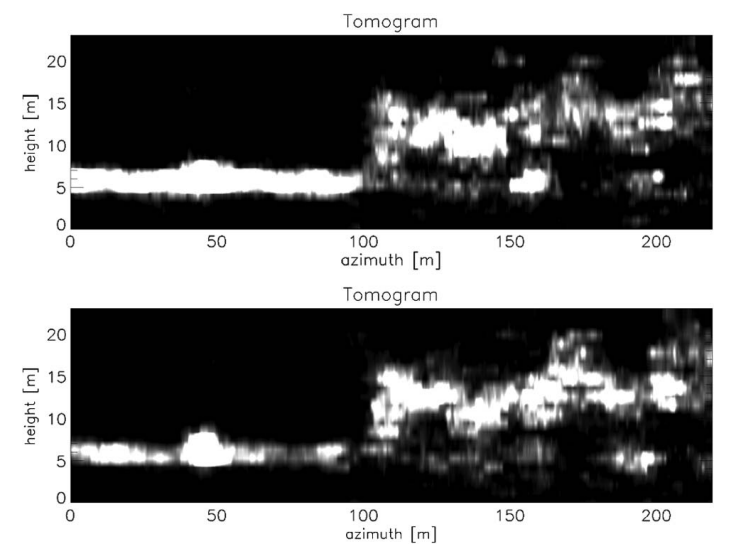

Fig. 6. Tomograms of profile 1 related to the two trucks. (Top) VV polarization. (Bottom) HV polarization.

tomogram, scatterers characterized by the same ground-range coordinate are resolved in the vertical axis. The spot indicated by the ellipse corresponds to the hidden truck that, due to the track width, can be also identified in this direction. It should be noted that the existence of the vegetation above the target is due to the fact that several neighboring pixels have been used to build the covariance matrix.

In Fig. 5, the tomogram related to the container is illustrated. The container and the canopy are visible. Due to the fact that the contribution of the container can be already seen in the SLC images, the following analysis will focus on the hidden truck.

\section{B. Polarimetric Comparison}

In this section, the impact of polarization on SARTom will be analyzed. Due to the presence of different kinds of scattering mechanisms (e.g., natural and man-made), polarimetry is useful to extract the target contribution [13]. First, the tomographic results will be presented in the lexicographic (HH-HV-VV) basis.

Fig. 6 presents the azimuth profile containing the trucks. Comparing Fig. 3 with Fig. 6 (top), it is clear that the $\mathrm{HH}$ and VV polarizations yield similar results. Turning to the crosspolarized channel [see Fig. 6 (bottom)], it is apparent that the target contribution disappeared, probably due to the higher sensitivity of the HV channel to volumetric structures, which does not permit the reception of a backscattered signal from the target with significant power.

Let us now consider the Pauli decomposition that allows the first direct interpretation of the scattering mechanisms. In order to generate tomograms in the Pauli basis, the SLC images related to different linear polarization channels were first transformed to the Pauli basis before tomographic processing. The

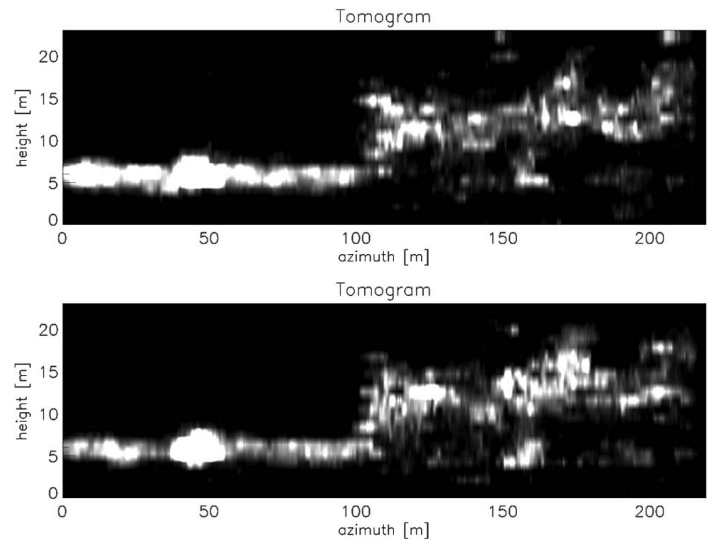

Fig. 7. Tomograms of the profile related to the two trucks in the Pauli basis. (Top) $P_{1}$. (Bottom) $P_{2}$.

well-known form of the monostatic scattering vector related to this basis is

$$
\begin{aligned}
\vec{k}_{3 P} & =\left[S_{\mathrm{hh}}+S_{\mathrm{vv}}, S_{\mathrm{hh}}-S_{\mathrm{vv}}, 2 S_{\mathrm{hv}}\right]^{T} / \sqrt{2} \\
& =\left[P_{1}, P_{2}, P_{3}\right]^{T}
\end{aligned}
$$

where $S_{i j}$ corresponds to the SLC image in the $i j$ polarization. In this basis, the first and second channels emphasize the oddand even-bounce contributions, respectively. As shown in the lexicographic basis, the third channel is related to volumetric contributions.

The tomograms representing the first and second elements of the Pauli basis are illustrated in Fig. 7. It is possible to observe that, for this target, the use of this basis extracts some features that are not apparent in the lexicographic one. For the truck under foliage, the Pauli ${ }_{1}\left(P_{1}\right)$ response is stronger for the rear part of the truck itself, while the Pauli $_{2}\left(P_{2}\right)$ component has a stronger answer for the front part of it. For the Pauli ${ }_{3}\left(P_{3}\right)$ contribution, refer to Fig. 6 (bottom).

\section{Coherent Linear Beamforming}

Usually, superresolution methods are not used to completely characterize polarimetric signatures because their response is more an indication of the scatterer position rather than a measure of its backscattered power. However, the polarimetric information can be partially recovered even for such methods by estimating the ellipticity and the orientation angle of the polarimetric ellipse [14]. For a direct interpretation of the scattering mechanisms, it is necessary to make use of a coherent beamformer [15], which, despite its reduced resolution, high sidelobe level, and ambiguity rejection characteristics, makes it possible to form the tomograms directly and yields meaningful amplitude and phase information. For the coherent beamformer, the tomograms can be presented in a color-coded image that allows one to identify the main scattering mechanisms.

The Pauli tomograms of the trucks are illustrated in Fig. 8. It is possible to note that the double-bounce contribution is the main reflection for the target outside the forest. For the hidden truck, the polarimetric representation allows for its detection because the Pauli ${ }_{3}$ channel is filtered out by the vegetation, and it is not present beneath the canopy (the truck corresponds to the region marked with the ellipse).

In order to compare Fig. 8 with the reconstructions obtained by means of the Capon beamformer and to image the ground on 


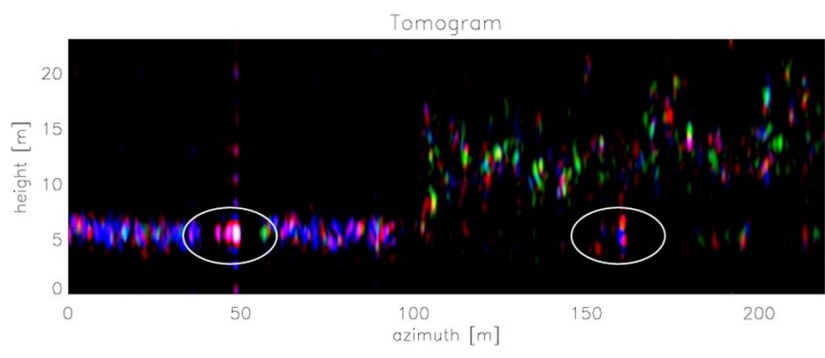

Fig. 8. PolSARTom reconstruction of the trucks in the Pauli basis by means of the coherent beamformer. The response is normalized to the total power. Coding: $\mathrm{BRG}\left(P_{1}, P_{2}\right.$, and $\left.P_{3}\right)$.

the truck outside the forest, despite its reduced backscattered power, normalization on a pixel-by-pixel basis with the total power along the multibaseline array was carried out [16]. Operating on the coherent beamforming response in this fashion, one compensates for the absence of the self-cancellation phenomenon that homogenizes reflectivity and is characteristic of the Capon beamforming. The dynamic range has been set to reflect the hidden target and the vegetation contribution. Checking the scattering mechanism of the ground outside the forest, it turned out that it could be consistently retrieved due to the dominance of the single-bounce reflection.

It should be noted that the improved estimation of the azimuth dimension of the target, when compared to the tomograms obtained by means of the Capon beamformer, is due to the fact that, for the coherent beamformer, there is no need to compute the sample covariance matrix. Therefore, the typical spreading effect of the Capon beamformer is not present, allowing one to estimate the length of the targets as 7-8 $\mathrm{m}$ with an actual length of $7 \mathrm{~m}$.

In principle, the reconstruction could be further improved by means of regularized linear inversion techniques that exploit the singular value decomposition. For a comparison of such techniques and the adaptive Capon beamforming, see [16].

\section{Truck Height Estimation}

Analyzing the tomograms obtained by means of the Capon beamforming at different polarizations and taking the average of the estimated power values along the azimuth interval of the target response, backscattering profiles of the truck, as shown in Fig. 9, can be obtained.

One can identify distinct contributions to the averaged profiles corresponding to the hidden truck and the vegetation. Therefore, by determining the bounds of these regions, one obtains an estimate of the truck height and of the canopy depth.

This analysis shows how, by simple exploitation of the polarimetric space, one can improve the visual estimation of the extremes of the boundary regions. The red dots indicate the boundary height of these two structures. The actual target height is $3 \mathrm{~m}$, and the estimated one is $2.3 \mathrm{~m}$. The bottom height of the truck is estimated from the HH polarization, whereas the top of it, for this particular test case, is estimated from the second Pauli component $\left(\mathrm{Pauli}_{2}\right)$.

The mismatch between the two measurements is due to the complex structure of the target. In practice, it is not possible to determine the location of the main phase centers (what the

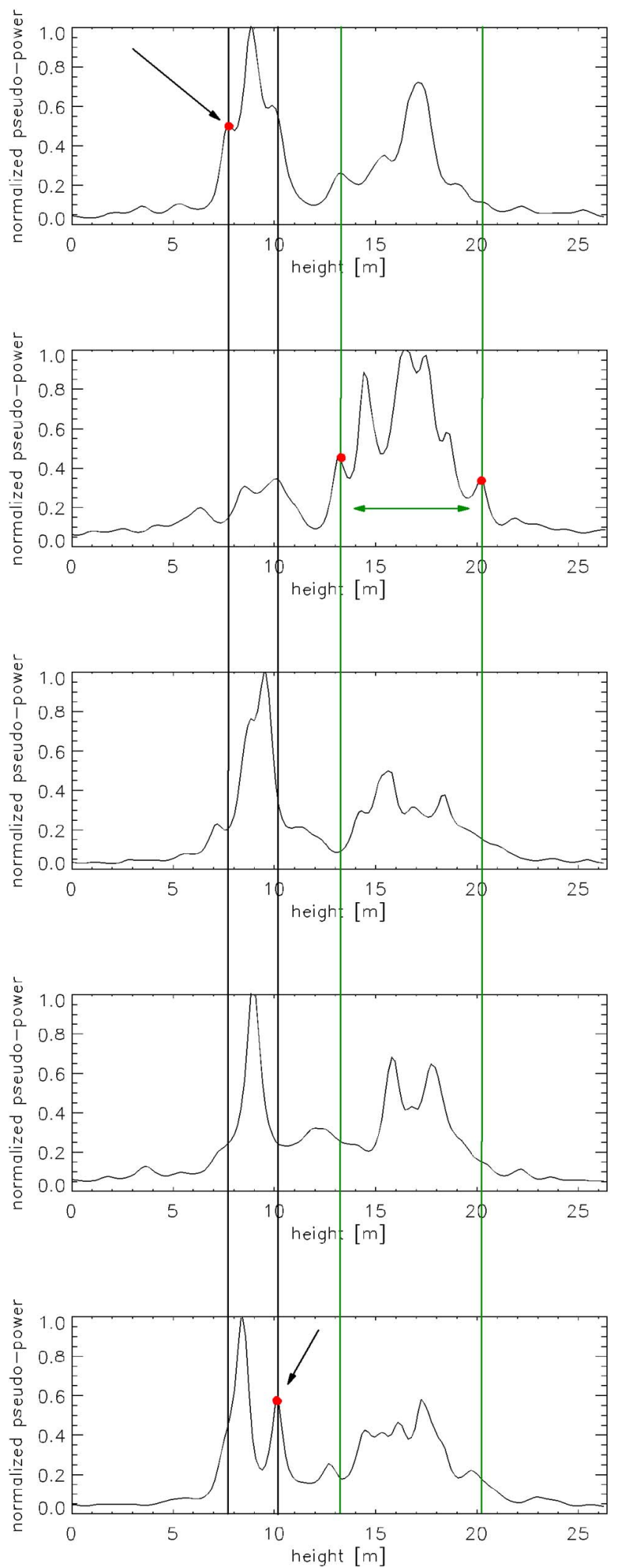

Fig. 9. SARTom profiling of the hidden truck obtained by means of the Capon method for different polarizations. (From top to bottom) HH, HV (Pauli3), VV, Pauli $_{1}$, and Pauli 2 . A truck height of $2.3 \mathrm{~m}$ and a canopy thickness of about $7 \mathrm{~m}$ are estimated.

sensor actually sees). However, the metallic body of the truck is raised from the ground because of the wheels; therefore, a slight underestimation is to be expected. 


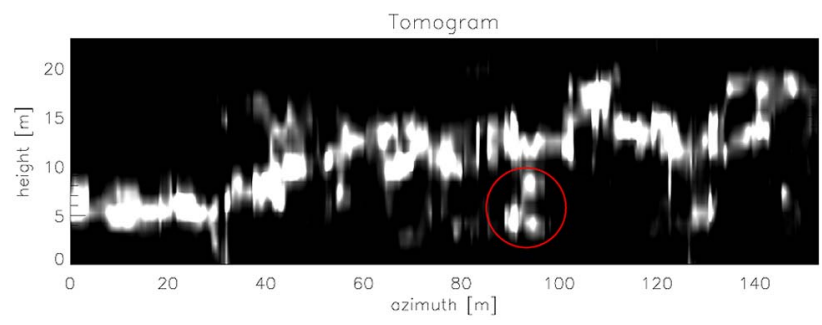

Fig. 10. Tomogram obtained by means of the MUSIC algorithm in the HH polarization and a tomographic constellation of $N=6$ tracks.

In order to verify the reliability of this analysis, the boundary height estimation has been also carried out for the canopy. As mentioned earlier, the polarimetric channel that is most sensitive to volumetric structures is the cross-polarized channel HV. Hence, this component should allow the best estimation of the canopy depth. Indeed, examining the HV averaged tomogram in Fig. 9, it is clear that the cross channel detects both the minimum and maximum heights of the canopy above the truck and, as expected, the boundary height can be determined just from this polarization channel in agreement with the theory. Finally, a canopy depth of $7 \mathrm{~m}$ was estimated. These results show that the combination of SARTom and polarimetry allows one to estimate the dimensions of the hidden target and its main backscattering mechanisms. The quality of the acquired airborne data resulting in an almost regular grid enables robust tomographic imaging in terms of scatterer separation without the need for any additional interpolation.

\section{Imaging With a Reduced Number OF ACQUISITIONS}

The main disadvantage of SARTom concerns the number of acquisitions required to perform reconstruction. On the one hand, a large tomographic aperture has to be spanned to obtain a reasonable resolution, whereas, on the other hand, a sufficient sampling along such an aperture (i.e., a large number of acquisitions) is required to avoid aliasing. A reduction in the number of passes is of fundamental importance in view of exploiting tomography in future spaceborne missions.

In [17], some first results of layover solution for a point scatterer scenario, carried out on dual-baseline single-pass data, have been presented. In [6], subspace-based methods such as the MUSIC algorithm have been exploited to reduce the number of acquisitions without impacting the quality of the reconstruction for distributed scatterers.

In this context, it is interesting to observe how, for the case of the hidden truck, a dominant deterministic contribution is present in comparison with the vegetation. This condition allows the MUSIC algorithm to image the target even with a few acquisitions. In Fig. 10, the HH tomogram imaging the hidden truck produced using only $N=6$ acquisitions is illustrated. The model order selection is adaptively done for each azimuth position. One can observe that the truck is represented with more than one phase center. The related tomographic aperture corresponds to $L_{\text {tomo }}=100 \mathrm{~m}$. Due to the reduced number of acquisitions, the related covariance matrix requires less snapshots to be full rank; therefore, azimuth broadening of the response of the truck is reduced when compared to the previous (full-constellation) reconstructions.

\section{CONCLUSION}

In this letter, first experimental results concerning 3-D imaging of targets beneath foliage have been presented. The use of the coherent beamformer allows one to fully exploit the polarimetric space and associate a signature with the target in order to detect it. It has been shown that the polarimetric diversity can be also exploited in the case of superresolution methods to estimate the height of the hidden target. Moreover, due to the deterministic nature of its response, one can image the hidden target even with fewer acquisitions, as shown in Section V.

\section{ACKNOWLEDGMENT}

The authors would like to thank eOsphere Ltd. and the Electro-Magnetic Remote Sensing Defence Technology Centre for their support of this work, as well as the E-SAR team of DLR for their efforts in conducting the campaign. Moreover, the authors would like to thank the reviewers for their constructive comments.

\section{REFERENCES}

[1] A. Reigber and A. Moreira, "First demonstration of airborne SAR tomography using multibaseline L-band data," IEEE Trans. Geosci. Remote Sens., vol. 38, no. 5, pp. 2142-2152, Sep. 2000.

[2] G. Fornaro, F. Serafino, and F. Soldovieri, "Three-dimensional focusing with multipass SAR data," IEEE Trans. Geosci. Remote Sens., vol. 41, no. 3, pp. 507-517, Mar. 2003.

[3] J. Capon, "High-resolution frequency-wavenumber spectrum analysis," Proc. IEEE, vol. 57, no. 8, pp. 1408-1418, Aug. 1969.

[4] R. Schmidt, "Multiple emitter location and signal parameter estimation," IEEE Trans. Antennas Propag., vol. AP-34, no. 3, pp. 276-280, Mar. 1986.

[5] F. Lombardini and A. Reigber, "Adaptive spectral estimation for multibaseline SAR Tomography with airborne L-band data," in Proc. IGARSS, Toulouse, France, Jul. 2003, pp. 2014-2016.

[6] M. Nannini, R. Scheiber, and A. Moreira, "Estimation of the minimum number of tracks for SAR tomography," IEEE Trans. Geosci. Remote Sens., vol. 47, no. 2, pp. 531-543, Feb. 2009.

[7] O. Frey, F. Morsdorf, and E. Meier, "Tomographic imaging of a forested area by airborne multi-baseline P-band SAR," Sensors, vol. 8, no. 9, pp. 5884-5896, Sep. 2008.

[8] S. Tebaldini, "Single and multipolarimetric SAR tomography of forested areas: A parametric approach," IEEE Trans. Geosci. Remote Sens., vol. 48, no. 5, pp. 2375-2387, May 2010.

[9] S. Cloude, D. Corr, and M. Williams, "Target detection beneath foliage using polarimetric synthetic aperture radar interferometry," Waves Random Complex Media, vol. 14, no. 2, pp. 393-414, Mar. 2004.

[10] A. Moreira, J. Mittermayer, and R. Scheiber, "Extended chirp scaling algorithm for air- and spaceborne SAR data processing in stripmap and ScanSAR imaging modes," IEEE Trans. Geosci. Remote Sens., vol. 34, no. 5, pp. 1123-1136, Sep. 1996.

[11] P. Prats, K. A. Camara de Macedo, A. Reigber, R. Scheiber, and J. J. Mallorqui, "Comparison of topography- and aperture-dependent motion compensation algorithms for airborne SAR," IEEE Geosci. Remote Sens. Lett., vol. 4, no. 3, pp. 349-353, Jul. 2007.

[12] M. Nannini and R. Scheiber, "Height dependent motion compensation and coregistration for airborne SAR tomography," in Proc. IGARSS, Barcelona, Spain, Jul. 2007, pp. 5041-5044.

[13] J. S. Lee and E. Pottier, Polarimetric Radar Imaging: From Basics to Applications. Boca Raton, FL: CRC Press, 2009.

[14] S. Guillaso and A. Reigber, "Polarimetric SAR tomography," in Proc. POLINSAR, Frascati, Italy, Jan. 2005.

[15] M. Nannini and R. Scheiber, "A time domain beamforming algorithm for SAR tomography," in Proc. EUSAR, Dresden, Germany, May 2006.

[16] F. Lombardini, M. Pardini, G. Fornaro, F. Serafino, L. Verrazzani, and M. Costantini, "Linear and adaptive spaceborne three-dimensional SAR tomography: A comparison on real data," IET Radar, Sonar Navig., vol. 3, no. 4, pp. 424-436, Aug. 2009.

[17] F. Lombardini, J. Ender, L. RoBing, M. Galletto, and L. Verrazzani, "Experiments of interferometric layover solution with the three-antenna airborne AER-II SAR system," in Proc. IGARSS, Anchorage, AK, Sep. 2004, pp. 3341-3344. 